\title{
Magnetoencephalography and Auditory Neural Representations
}

\author{
J.Z. Simon ${ }^{1,2}$ and N. Ding ${ }^{1}$ \\ ${ }^{1}$ Department of Electrical \& Computer Engineering, University of Maryland, College Park MD 20815, USA \\ ${ }^{2}$ Department of Biology, University of Maryland, College Park MD 20815, USA
}

\begin{abstract}
Complex sounds, especially natural sounds, can be parametrically characterized by many acoustic and perceptual features, one among which is temporal modulation. Temporal modulations describe changes of a sound in amplitude (amplitude modulation, $\mathrm{AM}$ ) or in frequency (frequency modulation, FM). AM and FM are fundamental components of communication sounds, such as human speech and speciesspecific vocalizations, as well as music. Temporal modulations are encoded in at least two ways, temporal coding and rate coding. Magnetoencephalography (MEG), with its high temporal resolution and simultaneous access to multiple auditory cortical areas, is a non-invasive tool that can measure and describe the temporal coding of auditory modulations. We refer to the neural temporal encoding of temporal acoustic modulations as "modulation encoding". For simple, individually presented, acoustic modulations, modulation encoding is well described by a simple modulation transfer function (MTF). Even in this simple case, however, the MTF may depend strongly on the type of modulation being encoded (e.g. AM vs. FM, narrowband vs. broadband) or the context in which the modulation is heard (e.g. attended vs. unattended). Here we present a range of different types of modulation encoding employed by human auditory cortex. The simplest examples are for sinusoidally amplitude modulated carriers of a range of bandwidths (with special emphasis on those modulation rates relevant to speech and other natural sounds: below a few tens of $\mathrm{Hz}$ ). We provide evidence that the modulation transfer functions are lowpass in shape and relatively independent of bandwidth. When several modulations are applied concurrently however, the modulation encoding typically, but not always, becomes non-linear: the auditory modulations are at the rates of the acoustic modulations but also at the rates of cross-modulation frequencies. The physiological occurrence, or not, of these cross terms seem be in accord with the psychophysical concept of modulation filterbanks.
\end{abstract}

Keywords-Magnetoencephalography, cortex, modulations.

\section{INTRODUCTION}

Speech and environmental sounds contain slow modulations in both amplitude (AM) and frequency (FM). Speech intelligibility depends strongly on the integrity of modulations within a range of perceptually-relevant rates, the same range which drives cortical responses most vigorously as measured by single-unit activity. The neural mechanisms by which this modulation is encoded are important to our understanding of perception, and to applications such as auditory prostheses. Speech and natural sounds contain simultaneous AM and FM, of various rates and embedded in other sounds, so it is crucial to determine the encoding of compound and noisy modulations as well as simple ones.

In humans, neurophysiological studies of temporal methods in auditory coding are generally limited to non-invasive techniques. For these studies, MEG is an appropriate tool, as it has excellent temporal resolution, is silent, and can localize neural sources with suitable resolution (particularly so in auditory areas).

\section{A. Modulation Sensitivity}

Psychophysically, human listeners attending to spectrally simple broadband sounds are most sensitive to amplitude modulations at a few $\mathrm{Hz}$, peaking near $10 \mathrm{~Hz}$ and diminishing substantially by $100 \mathrm{~Hz}$ [1]. This result also holds when increasing the spectral complexity of the carrier (see, e.g. $[2,3]$ ). Speech intelligibility is strongly dependent on modulation rates below $20 \mathrm{~Hz}$, for both amplitude modulation $[4,5]$ and frequency modulation components of speech [6].

\section{B. MEG and Temporal Response Properties}

Complementary to hemodynamic methods (e.g. fMRI), MEG is more limited in its spatial resolution but virtually unlimited in its temporal resolution (e.g. down to $\sim 1 \mathrm{~ms}$ ). Since temporal aspects of auditory signals play such a crucial role in both perception and speech processing, MEG is a logical tool to employ for these purposes. Additionally, MEG is silent, unlike fMRI.

MEG measures the magnetic fields generated by neuronal current flow, using SQUID based detectors (Superconducting Quantum Interference Devices) to make highgain recordings [7]. It is believed the main source for MEG is current flow in pyramidal cells' apical dendrites. [7]

A major benefit over EEG (which measures electric potentials generated by the same neural activity) is high sensitivity to activity within auditory cortex (where pyramidal cell dendrites are parallel to the surface). In contrast, EEG responses mix across cortical hemispheres and are strongest medially (see, e.g., [8]). Another benefit to MEG is that results from data averaged over subjects are typically visible in individual subjects as well. K.E. Herold, W.E. Bentley, and J. Vossoughi (Eds.): SBEC 2010, IFMBE Proceedings 32, pp. $45-48,2010$.
www.springerlink.com 
The auditory steady state response (SSR) is the neural response to a stationary acoustic stimulus. SSR responses are a rich source of neurophysiological data that have strong potential to make compelling links with animal-based cortical physiology. Auditory SSR latencies from MEG, typically 30-40 ms $[9,10]$, are much better matched to latencies seen in single neuron studies than the M100 or even M50.

SSR was first measured in humans by using EEG, where the strongest response is typically found at $40 \mathrm{~Hz}$ [11]. Rates below $20 \mathrm{~Hz}$ are less well explored due the lower signal to noise ratio (SNR) compared to near $40 \mathrm{~Hz}$. Using MEG, Ross et al. [9] found a slowly decreasing plateau from $20 \mathrm{~Hz}$ down to $10 \mathrm{~Hz}$, but with increasing error bars. EEG studies give conflicting results, but, with care in rejecting strong but statistically non-significant responses, Picton et al. [8] found a trend that the EEG response increases as rate decreases below $20 \mathrm{~Hz}$. Our MEG results support this result in much greater detail.

\section{Methods}

15 subjects ( 9 female) (Experiment 1$)$, and 10 subjects ( 5 female) (Experiment 2), all right handed [12], who reported normal hearing and no history of neurological disorder, listened to the acoustic stimuli while MEG recordings were taken. The procedures were approved by the University of Maryland institutional review board and written informed consent was obtained from each participant. Subjects were paid for their participation.

In Experiment 1, sinusoidally amplitude-modulated sounds of $2000 \mathrm{~ms}$ duration were presented to each subject. Twenty stimuli were made with 5 modulation frequencies $(1.5 \mathrm{~Hz}, 3.5 \mathrm{~Hz}, 7.5 \mathrm{~Hz} 15.5 \mathrm{~Hz}$ and $31.5 \mathrm{~Hz})$ and 4 different carriers (pure tone at $707 \mathrm{~Hz} ; 1 / 3$ octave pink noise; 1 octave pink noise $\mathrm{Hz}$ and 5 octave pink noise (all centered at $707 \mathrm{~Hz}$ ) with $100 \%$ modulation depth. The four different bandwidth conditions are henceforth referred to as $0,1 / 3,1$, and 5 octave bandwidths.

In Experiment 2, the stimulus is a tone simultaneously frequency and amplitude modulated. The FM modulation frequency, $f_{F M}$, is fixed at $37.7 \mathrm{~Hz}$. The maximum frequency deviation from the base carrier frequency, $550 \mathrm{~Hz}$, is 330 $\mathrm{Hz}$. The AM modulation frequency, $f_{A M}$, is varied, taking on values of $0.3,0.7,1.7,3.1,4.9,9.0$, and $13.8 \mathrm{~Hz}$. The specific values of $f_{F M}$ and $f_{A M}$ are selected to avoid harmonic overlaps. Stimulus duration is $21 \mathrm{~s}$.

Subjects were placed horizontally in a dimly lit magnetically shielded room (Yokogawa Electric Corporation, Tokyo). The signals were delivered to the subjects' ears with $50 \Omega$ sound tubing (E-A-RTONE 3A, Etymotic Research, Inc), attached to E-A-RLINK foam plugs inserted into the ear-canal and presented binaurally at a comfortable loudness of approximately $70 \mathrm{~dB}$ SPL.

MEG recordings were conducted using a 157-channel axial gradiometer whole-head system (Kanazawa Institute of Technology, Kanazawa, Japan). Its detection coils form a uniform array on a helmet-shaped surface of the dewar bottom, with about $25 \mathrm{~mm}$ between the centers of two adjacent $15.5 \mathrm{~mm}$ diameter coils. Sensors are axial gradiometers with $50 \mathrm{~mm}$ baseline; field sensitivities are $5 \mathrm{fT} / \sqrt{\mathrm{Hz}}$ or better in the white noise region. Three magnetometers measure the environmental magnetic field. The signals were bandpassed between 0 or $1 \mathrm{~Hz}$ and $200 \mathrm{~Hz}$, notch filtered at $60 \mathrm{~Hz}$, and sampled at the rate of $500 \mathrm{~Hz}$ or $1 \mathrm{kHz}$. The $1 \mathrm{~Hz}$ high pass filter's influence on the amplitude and phase of MEG recordings is corrected.

Two denoising techniques were applied off-line: TSPCA [13], to remove external noise (filtered versions of the reference signals), and SNS [14], to remove noise internal to individual gradiometers. TS-PCA used a $\pm 100 \mathrm{~ms}$ range of filter taps; SNS used 10 channel neighbors. Finally, DSS [15] a blind source separation technique was applied to preserve phase-locked neural activities. The DSS components are sorted based on how much of the response power is phase-locked to the stimulus. Only the first component is kept for further analysis in this study.

A single current dipole modeled the auditory response in each hemisphere. The lead field was calculated using the complex version of the Sarvas spherical head model [16]. A isotropic sphere model was build for each subject, using MEG Laboratory 2.001M (Yokogawa Electric Corporation, Tokyo). The dipole moment is estimated using least squares [17] while the dipole position is estimated using a modified simplex search with clustering [18].

\section{Results}

\section{A. Experiment 1}

The relation between the strength of neural AM response and the stimulus $f_{A M}$ is commonly known as modulation transfer function (MTF). Dipole strengths of MEG responses are shown in Fig. 1 as a function of the stimulus AM rate and carrier bandwidth, for each hemisphere. This low-pass pattern is also seen in intracranial recordings [19]. The MEG response power in the right hemisphere is stronger than that in the left hemisphere for $1.5 \mathrm{~Hz}$ AM with pure tone carrier and $31.5 \mathrm{~Hz}$ AM with all carriers (paired ttest, $t(13)>2.2, p<0.05)$. The MEG response power is significantly affected by the stimulus AM rate (2-way ANOVA, $F(4,279)>22, p<10^{-4}$ for both hemispheres) but not the stimulus bandwidth. There is a significant interaction between the effect of AM rate and the effect of carrier 

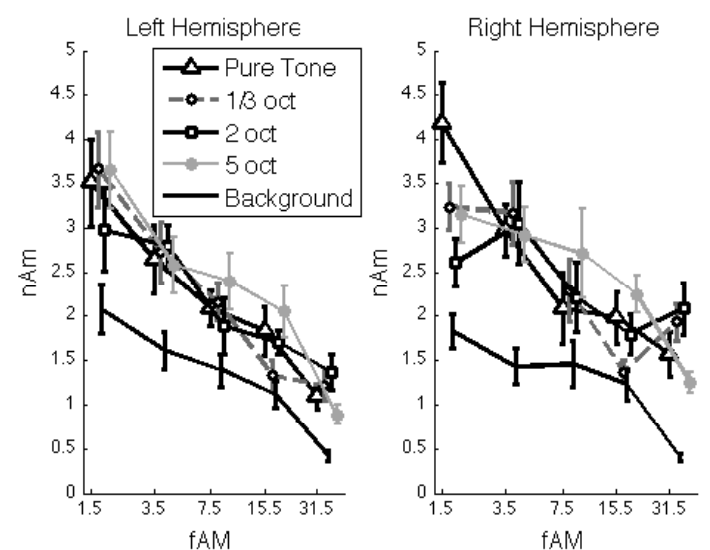

Fig. 1 Dipole strengths of MEG responses averaged over subjects. Error bars are standard error over subjects

bandwidth $(F(12,279)>2.04, p<0.03)$ in the right hemisphere.

\section{B. Experiment 2}

An MEG response at the stimulus $f_{A M}$ is observed in all stimulus conditions.. The MTF measured by the power of the MEG response at $f_{A M}$ has a low-pass pattern: the power of the MEG response to an AM sound decreases with increasing $f_{A M}$ of that sound. It needs to be clarified, however, whether the low-pass pattern of the MTF results from stimulus-driven SSR or background noise. One can estimate the power of stimulus-driven SSR by subtracting the estimated power of background noise at $f_{A M}$ from the power of measured MEG signal at $f_{A M}$. The MTF measured by this corrected power of the SSR at $f_{A M}$ still shows a low-pass pattern and can be modeled as a linear function of $f_{A M}$ measured in $\mathrm{Hz}$ (Fig. 2). The slope of the fitted linear function is $-0.96 \mathrm{~dB} / \mathrm{Hz}$ (99\% confidence interval, -1.17 to -0.74 $\mathrm{dB} / \mathrm{Hz})$. For $f_{A M}$ higher than $1 \mathrm{~Hz}$, the slope of the MTF can also be fitted as $-3.6 \mathrm{~dB} /$ oct ( $99 \%$ confidence interval, -4.8 to $-2.5 \mathrm{~dB} /$ oct $)$. Since the slope of the fitted line is significantly negative $(p<0.01)$, the low-pass pattern of the MTF is statistically significant for $f_{A M}$ lower than $15 \mathrm{~Hz}$. To reduce subject-to-subject variability, the corrected power is normalized before the regression analysis. Even without any correction or normalization, the slope of the MTF is still significantly negative $(99 \%$ confidence interval, -1.73 $\mathrm{dB} / \mathrm{Hz}$ to $-0.29 \mathrm{~dB} / \mathrm{Hz}$ ). To investigate whether the reduction in the evoked power of the MEG response at $f_{A M}$ is due to a loss of energy in every single trial or a loss of phase locking over trials, we calculated the phase coherence value [20] of the MEG response at $f_{A M}$ over trials. One way ANOVA shows the phase coherence values does not significantly change when the stimulus $f_{A M}$ increases from 0.7 $\mathrm{Hz}$ to $13.8 \mathrm{~Hz}(F(5,54)=0.84, p>0.5)$. Hence, the

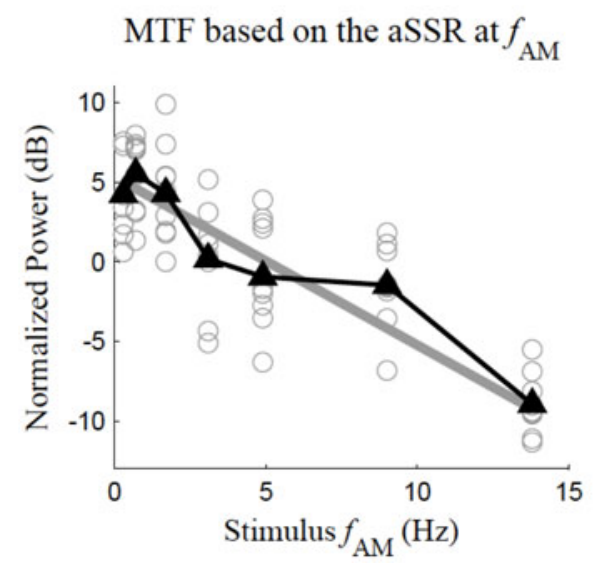

Fig. 2 Analysis of the power of the SSR at $f_{A M}$. The MTF calculated as a function of the corrected power of the SSR at $f_{A M}$ and stimulus $f_{A M}$. Each gray hollow circle represents the corrected power of the SSR at $f_{A M}$ for one subject. The black line marked by triangles shows the grand averaged MTF. The gray line is the optimal linear fit of the MTF

low-pass pattern of evoked power of SSR at $f_{A M}$ is due to a change in single trial power rather than a change in over trial phase coherence. Since both the neural response power and background noise power are strongest at low frequencies, regression analysis was used to show that the signal to noise ratio of the neural response at $f_{A M}$ does not significantly increase or decrease when $f_{A M}$ increases $(p>0.6)$. If the neural response power at $f_{A M}, 2 f_{A M}$ and $3 f_{A M}$ are combined, the MTF has a slope of $-1.06 \mathrm{~dB} / \mathrm{Hz}(99 \%$ confidence interval, $-1.30 \mathrm{~dB} / \mathrm{Hz}$ to $-0.82 \mathrm{~dB} / \mathrm{Hz}$ ).

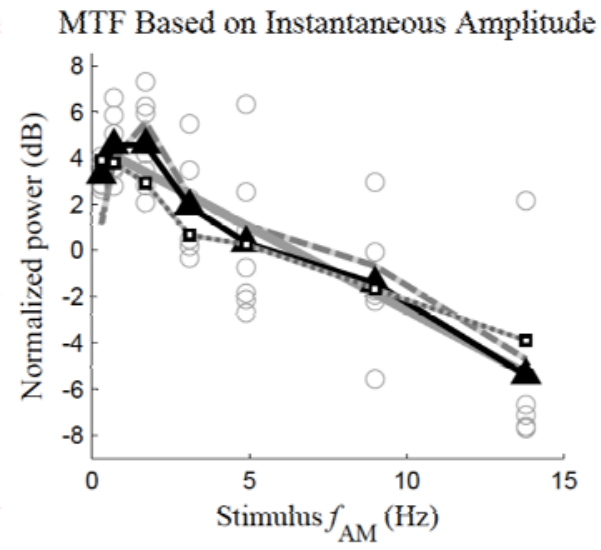

Fig. 3 Analysis of the instantaneous amplitude of the SSR at $f_{F M}$. The solid black line with triangle markers is the MTF averaged over all subjects. The solid gray line is the optimal linear fit of the MTF while the dotted gray line with white square markers is the MTF predicted a model. The power of the instantaneous amplitude for each subject and each condition is shown as a gray hollow circle. The instantaneous amplitude's power at $f_{A M}$ is plotted as the dashed gray line 
One of the primary goals of this work is to examine the interaction between fast modulations and slow modulations. Since the instantaneous amplitude of the SSR at $f_{F M}$ oscillates with fundamental frequency $f_{A M}$, it is a neural correlate of the stimulus slow AM. Consequently, the relation between the power of the instantaneous amplitude and the stimulus $f_{A M}$ can also be regarded as an effective MTF. We estimate the power of the instantaneous amplitude as the sum of the power at the first four harmonics of $f_{A M}$. This MTF (Fig. 3) has a slope of $-0.72 \mathrm{~dB} / \mathrm{Hz}(99 \%$ confidence interval, -0.95 to $-0.49 \mathrm{~dB} / \mathrm{Hz}$ ). When $f_{A M}$ is higher than 1 $\mathrm{Hz}$, the slope of the MTF can also be fitted as $-3.0 \mathrm{~dB} /$ oct ( $99 \%$ confidence interval, -5.0 to $-1.6 \mathrm{~dB} / \mathrm{oct}$ ). For this MTF calculation, the power at each harmonic of $f_{A M}$ was corrected by subtracting the power of background noise at that frequency; the estimate of the power of the instantaneous amplitude is also normalized to reduce subject-to-subject variability Without any correction or normalization, the MTF slope is still significantly negative $(p<0.01)$.

As the stimulus $f_{A M}$ increases, the power of the MEG response at $f_{F M}$ decreases at $0.86 \mathrm{~dB} /$ oct while the power of the instantaneous amplitude of the SSR at $f_{F M}$ decreases 3.0 $\mathrm{dB} /$ oct. If the SSR at $f_{F M}$ is assumed to be sinusoidally amplitude modulated, the neural AM modulation depth of the SSR can be estimated based on the ratio between the power of the instantaneous amplitude of the SSR and the power of the MEG response at $f_{F M}$. Hence, with the sinusoidal AM assumption, the neural AM modulation depth should decreases at $2.1 \mathrm{~dB} /$ oct.

\section{Conclusions}

First, this study characterizes the properties of MEG responses to AM below $30 \mathrm{~Hz}$. The SSR is strongest at the lowest modulation rates and decreases $2-4 \mathrm{~dB}$ per octave. For jointly modulated stimuli, the instantaneous amplitude of the SSR at $f_{F M}$ also oscillates with fundamental frequency $f_{A M}$. Due to these neural interactions, the information in slow $\mathrm{AM}$ is simultaneously encoded in neural oscillations at $f_{A M}$ and $f_{F M}$.

\section{ACKNOwledgments}

We thank Max Ehrman and Jeff Walker for excellent technical support. This research was supported by the National Institutes of Health (NIH) grant R01DC008342.

\section{REFERENCES}

1. Viemeister NF (1979) Temporal modulation transfer functions based upon modulation thresholds. J Acoust Soc Am 66:1364-1380
2. van Zanten GA, Senten CJ (1983) Spectro-temporal modulation transfer function (STMTF) for various types of temporal modulation and a peak distance of $200 \mathrm{~Hz}$. J Acoust Soc Am 74:52-62

3. Chi T, Gao Y, Guyton MC, Ru P, Shamma S (1999) Spectro-temporal modulation transfer functions and speech intelligibility. J Acoust Soc Am 106:2719-2732

4. Steeneken HJ, Houtgast T (1980) A physical method for measuring speech-transmission quality. J Acoust Soc Am 67:318-326

5. Drullman R, Festen JM, Plomp R (1994) Effect of temporal envelope smearing on speech reception. J Acoust Soc Am 95:1053-1064

6. Zeng FG, Nie K, Stickney GS, Kong YY, Vongphoe M, Bhargave A, Wei C, Cao K (2005) Speech recognition with amplitude and frequency modulations. Proc Natl Acad Sci U S A 102:2293-2298

7. Hamalainen M, Hari R, Ilmoniemi RJ, Knuutila J, Lounasmaa OV (1993) Magnetoencephalography - Theory, Instrumentation, and Applications to Noninvasive Studies of the Working Human Brain. Reviews of Modern Physics 65:413-497

8. Picton TW, John MS, Dimitrijevic A, Purcell D (2003) Human auditory steady-state responses. Int J Audiol 42:177-219

9. Ross B, Borgmann C, Draganova R, Roberts LE, Pantev C (2000) A high-precision magnetoencephalographic study of human auditory steady-state responses to amplitude-modulated tones. J Acoust Soc Am 108:679-691

10. Schoonhoven R, Boden CJ, Verbunt JP, de Munck JC (2003) A whole head MEG study of the amplitude-modulation-following response: phase coherence, group delay and dipole source analysis. Clin Neurophysiol 114:2096-2106

11. Galambos R, Makeig S, Talmachoff PJ (1981) A 40-Hz auditory potential recorded from the human scalp. Proc Natl Acad Sci U S A 78:2643-2647

12. Oldfield RC (1971) The assessment and analysis of handedness: The Edinburgh inventory. Neuropsychologia 9 (1), 97-113

13. de Cheveigné A, Simon JZ (2007) Denoising based on time-shift PCA. J. Neurosci. Methods 165 (2), 297-305

14. de Cheveigné A, Simon JZ (2008a) Sensor noise suppression. J. Neurosci. Methods 168 (1), 195-202

15. de Cheveigné A, Simon JZ (2008b) Denoising based on spatial filtering. J. Neurosci. Methods 171 (2), 331-339

16. Sarvas J (1987) Basic mathematical and electromagnetic concepts of the biomagnetic inverse problem. Phys. Med. Biol. 32, 11-22

17. Mosher JC, Baillet S, Leahy RM (2003) Equivalence of linear approaches in bioelectromagnetic inverse solutions. IEEE Workshop on Statistical Signal Processing, St. Louis

18. Uutela K, Hamalainen M, Salmelin R (1998) Global optimization in the localization of neuromagnetic sources. IEEE Trans. Biomed. Eng. 45 (6), 716-723

19. Liegeois-Chauvel C, Lorenzi C, Trebuchon A, Regis J, Chauvel P (2004) Temporal Envelope Processing in the Human Left and Right Auditory Cortices. Cereb Cortex 14:731-740

20. Fisher NI (1993) Statistical analysis of circular data. Cambridge [England] ; New York, NY, USA: Cambridge University Press

\author{
Author: Jonathan Z. Simon \\ Institute: Electrical \& Computer Engineering \\ Street: University of Maryland \\ City: $\quad$ College Park, MD 20815 \\ Country: USA \\ Email: jzsimon@umd.edu
}

